\title{
CONCENTRAÇÃO DE METAIS EM SEDIMENTOS DA LAGOA DO PARQUE METROPOLITANO DE PITUAÇU, BAHIA/BRASIL
}

\author{
E. da SILVA ${ }^{1 *}$, N. F. A SOUZA², Z. C. V. VIANA, P. S. MARINHO ${ }^{3}$ e V. L. C. S. SANTOS $^{3}$ \\ ${ }^{1}$ Universidade Federal de Campina Grande - UFCG \\ ${ }^{2}$ Central de Tratamento de Efluentes Líquidos - CETREL - Bahia \\ ${ }^{3}$ Universidade Federal da Bahia - UFBA \\ edevaldos@yahoo.com.br
}

Submetido 02/05/2016 - Aceito 04/07/2017

DOI: $10.15628 /$ holos.2017.4502

\section{RESUMO}

A lagoa de Pituaçu está inserida no Parque Metropolitano de Pituaçu (PMP). Esta recebe efluentes domésticos e pluviais e encontra-se em um processo acelerado de degradação ambiental. O presente trabalho teve como objetivo determinar a concentração de metais ( $\mathrm{Al}, \mathrm{Cu}, \mathrm{Fe}, \mathrm{Mn}, \mathrm{V}$ ) em sedimentos superficiais da lagoa do Parque Metropolitano de Pituaçu, Salvador, Bahia, Brasil. Foram definidos 7 pontos amostrais. A determinação de metais ( $\mathrm{Al}, \mathrm{Cu}, \mathrm{Fe}, \mathrm{Mn}$ e $\mathrm{V}$ ) foi realizada utilizando a técnica da espectrometria de emissão óptica com plasma indutivamente acoplado (ICP OES). A faixa de concentrações encontradas para os metais foram: Al (3.618-27.210 mg kg-1); Fe (11.968-122.030 $\left.\mathrm{mg} \mathrm{kg}^{-1}\right) ; \mathrm{Mn}$ $\left(44,00-481,50 \mathrm{mg} \mathrm{kg}^{-1}\right) ; \mathrm{V}\left(25,00-111,50 \mathrm{mg} \mathrm{kg}^{-1}\right)$ e $\mathrm{Cu}$

(4,05-25,50 mg kg-1). A ordem de concentração nos diversos pontos foi $\mathrm{Fe}>\mathrm{Al}>\mathrm{Mn}>\mathrm{V}>\mathrm{Cu}$ ou $\mathrm{Fe}>\mathrm{Al}>\mathrm{Mn}=\mathrm{V}>\mathrm{Cu}$, com as maiores e as menores concentrações de todos os metais, encontradas nos pontos P1 e P3, respectivamente. Houve variações espaciais nas concentrações dos metais, com maiores concentrações nos pontos amostrais próximos de atividades antrópicas (efluentes e atividade agrícola). Esse estudo reporta níveis relativamente altos, especialmente para o $\mathrm{V}$, indicando possíveis necessidades de ampliar ações de preservação ou monitoramento ambiental para evitar a acumulação desses metais em níveis tóxicos para a água e para o sedimento.

PALAVRAS-CHAVE: Lagos, Contaminação antrópica, Efluente, ICP OES.

\section{METALS CONCENTRATION IN SEDIMENT LAGOON OF METROPOLITAN PARK PITUAÇU, BAHIA/BRAZIL}

\begin{abstract}
The Pituaçu lagoon is part of the Pituaçu Metropolitan Park (PMP). Its receives domestic wastewater and rainwater and is in an accelerated process of environmental degradation. This study aimed to determine the concentration of metals ( $\mathrm{Al}, \mathrm{Cu}, \mathrm{Fe}, \mathrm{Mn}$, $\mathrm{V})$ in pond sediments Pituaçu Metropolitan Park, Salvador, Brazil. Seven sampling points were defined. The determination of metals ( $\mathrm{Al}, \mathrm{Cu}, \mathrm{Fe}, \mathrm{Mn}$ and $\mathrm{V}$ ) was accomplished using the technique of optical emission spectrometry with inductively coupled plasma (ICP OES). The range of concentrations found were: $\mathrm{Al}(3,618-$ $\left.27,210 \mathrm{mg} \mathrm{kg}^{-1}\right)$; Fe (11,968-122,030 $\left.\mathrm{mg} \mathrm{kg}^{-1}\right)$; Mn (44.00 to $\left.481.50 \mathrm{mg} \mathrm{kg}^{-1}\right) ; \mathrm{V}\left(25.00\right.$ to $111.50 \mathrm{mg} \mathrm{kg}^{-1}$ ) and $\mathrm{Cu}$
\end{abstract}

(4.05 to $25.50 \mathrm{mg} \mathrm{kg}^{-1}$ ). The order of concentration at various points was $\mathrm{Fe}>\mathrm{Al}>\mathrm{Mn}>\mathrm{V}>\mathrm{Cu}$ or $\mathrm{Fe}>\mathrm{Al}>\mathrm{Mn}=\mathrm{V}>$ $\mathrm{Cu}$, with the highest and lowest concentrations of all metals found in the points $\mathrm{P} 1$ and $\mathrm{P} 3$, respectively. There was spatial variations in the concentrations of metals, with higher concentrations in sample points with anthropogenic activities (effluents and agricultural activity). This study reports relatively high levels, especially for $V$, indicating possible need of expand conservation actions or environmental monitoring to avoid accumulation of these metals in toxic levels for water, sediment and its biota.

KEYWORDS: Lakes, Anthropogenic contamination, Effluent, ICP OES. 


\section{INTRODUÇÃO}

As lagoas são componentes biológicos de ecossistemas caracterizadas como sistemas abertos contidos em compartimentos maiores denominados bacia hidrográfica (TUCCl, 1997). Esta bacia pode ser então considerada um ente sistêmico, por onde se realizam os balanços de entrada de água proveniente da chuva e saída através do exutório, permitindo que sejam delineadas bacias e sub-bacias, cuja interconexão se dá pelos sistemas hídricos (PORTO; PORTO, 2008). Para a ciência ambiental, a bacia hidrográfica contém o conceito de integração que para os estudos de problemas ambientais são fundamentais, pois contém informações físicas, biológicas e socioeconômicas (COUTO, 2011).

As águas residuais transportam uma quantidade apreciável de materiais poluentes, os quais, presentes nos sistemas aquáticos, podem ser transformados em inúmeras formas químicas e/ou se associarem por adsorção, complexação, precipitação e assimilação biológica a diferentes tipos de partículas (orgânicas e inorgânicas). O que termina resultando em diferentes níveis de mobilização, transporte e biodisponibilidade podendo prejudicar a qualidade das águas dos rios (AL-MUSHARAFI; MAHMOUD; AL-BAHRY, 2014).

O ambiente aquático tem sido continuamente contaminado com substâncias químicas tóxicas provenientes de indústrias, agriculturas e atividades domésticas, sendo a disponibilidade dos elementos contaminantes fortemente influenciada pela concentração de matéria orgânica dissolvida. Os metais, por exemplo, através do processo de quelação, formam complexos que se precipitam no sedimento (MONTANHA et al., 2011).

O sedimento constitui um relevante compartimento no metabolismo dos corpos hídricos e nele se depositam muitos compostos que, incorporados à essa fase sólida tornam-se menos biodisponíveis, pois na fase aquosa os compostos são mais passíveis de serem sequestrados pelos organismos (SCHRIKS et al., 2010). Contudo, o sedimento é um compartimento ativo que acumula o material disperso na coluna d'água e o reprocessa, podendo torná-lo novamente disponível em solução (SOUZA et al., 2015).

Pode-se considerar o sedimento como um resumo das substâncias despejadas nos corpos hídricos, seja elas de origem natural ou geradas por diferentes atividades antrópicas. $O$ estudo da sua composição e/ou distribuição vertical pode permitir o conhecimento da atividade geoquímica local, bem como dos processos de sedimentação ocorridos (GONZÁLEZ et al., 2004) recente ou remotamente, além da quantificação das espécies contaminantes.

Os contaminantes tóxicos presentes no sedimento são potencialmente prejudiciais, relacionando-se à continuidade da degradação ambiental, mesmo quando presentes em baixos níveis na coluna d'água e ainda depois de cessada a descarga de poluentes no corpo d'água receptor (USEPA, 1997). Por se tratar de uma fonte acumuladora e repositora de contaminantes, nos sedimentos devem ser evitados procedimentos de manejo e outras interferências que impliquem na sua ressuspensão e forte oxigenação, a fim de evitar o risco de disponibilizar novamente os metais dissolvidos para a coluna de água (POMPÊO et al., 2013).

Apesar dos metais pesados também serem de fontes naturais (FIDAN et al., 2008), as suas principais fontes são de atividades antrópicas, principalmente de atividades agrícola, de mineração e da queima de combustíveis fósseis. Os riscos de contaminação dos ecossistemas aquáticos por metais pesados têm preocupado devido as suas consequências ecológicas (MEUCCl et al., 2009). A 
disponibilidade desses poluentes no sedimento pode causar stress ambiental na biota e sua retenção no ambiente pode favorecer uma provável bioacumulação (DURAL et al., 2007).

Uma avaliação da variação espacial da deposição de metais em sedimentos pode fornecer informações valiosas a respeito de sua origem e dos mecanismos de transporte, permitindo que se faça uma estimativa do impacto desses metais no meio ambiente e na biota aquática (SOUZA et al., 2015).

Ao longo de muitos anos, a Lagoa de Pituaçu tem recebido efluentes domésticos, industriais e pluviais. A ocupação na área do entorno da Bacia hidrográfica (Pituaçu) que o PMP está inserido, é tipicamente residencial de baixa renda, predominando a urbanização não planejada. Dessa forma o reservatório de Pituaçu encontra-se em um processo acelerado de degradação ambiental. Contudo, não se verificam pesquisas que reportem dados sobre as suas características geoquímicas.

Diante desta realidade, o objetivo dessa pesquisa é avaliar a concentração de metais (Al, $\mathrm{Cu}, \mathrm{Fe}, \mathrm{Mn}, \mathrm{V}$ ) em sedimentos da lagoa do Parque Metropolitano de Pituaçu, Bahia/BR e, com isso, colaborar com dados quantitativos que contribuam para a tomada de decisão em ações de mitigação ambiental para a preservação da lagoa.

\section{METODOLOGIA}

\section{1. Área de estudo}

A Bacia de Pituaçu (12056'37"S 38025'42"W) está localizada na Região Metropolitana de Salvador, Bahia, sendo formada pelo Rio Pituaçu e Rio Cachoeirinha. Nesta Bacia, encontra-se inserido o Parque Metropolitano de Pituaçu (PMP), como pode ser observado na Figura 1.

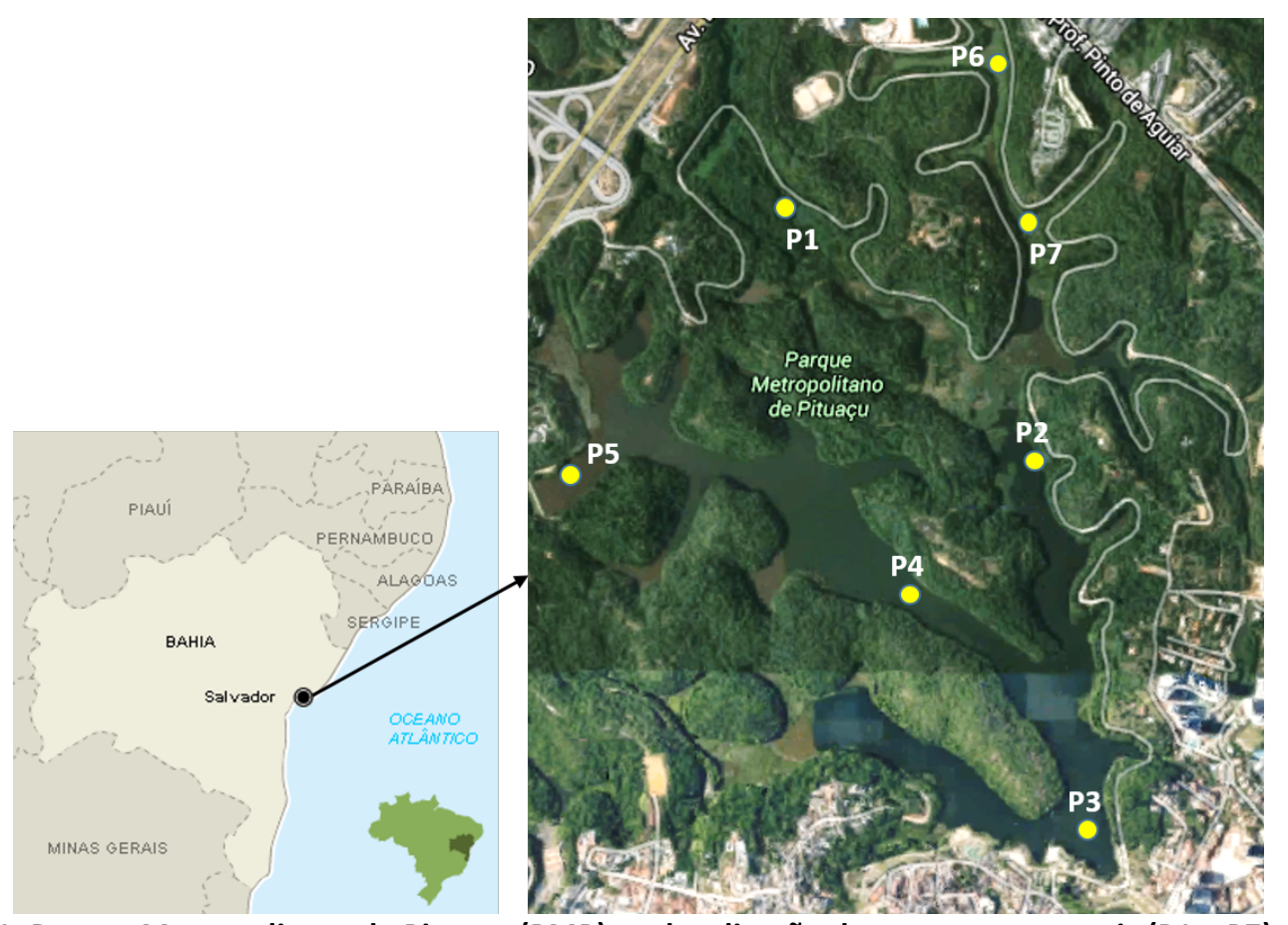

Figura 1: Parque Metropolitano de Pituaçu (PMP) e a localização dos pontos amostrais (P1 a P7) na Lagoa de Pituaçu. 
A lagoa de Pituaçu possui aproximadamente 660 ha. Parte do PMP, se assemelha ao formato de um trevo, com área total de $200.000 \mathrm{~m}^{2}$ e capacidade para 3 milhões de metros cúbicos de água, sendo circundada por uma ciclovia de $18 \mathrm{~km}$ de extensão (SEMA, 2015). O PMP tem sido considerado uma das mais importantes áreas de preservação ambiental e lazer de Salvador, um dos últimos fragmentos da Mata Atlântica e refúgio da fauna, que foi criado pelo Decreto no 23.666/77 do executivo estadual (SANTANA, 2003; SEMA, 2015).

Apesar desta lagoa estar inserida em uma área de preservação ambiental pode-se perceber nela sinais de poluição, possivelmente decorrentes de despejo de resíduos sólidos oriundos de atividades antrópicas relacionadas aos resíduos domésticos e industriais e à agricultura.

\subsection{Amostragem}

Inicialmente, foram realizadas visitas técnicas ao PMP para identificar e localizar geograficamente (Global Position System - GPS) os potenciais pontos de aporte antrópico considerados no presente estudo. Foram definidos 7 pontos amostrais (Tabela 1) ao longo da lagoa do PMP.

Tabela 1: Localização dos pontos amostrais

\begin{tabular}{|c|c|c|}
\hline Ponto amostral & \multicolumn{2}{|c|}{ Coordenadas geográficas } \\
\hline $\mathrm{P} 1$ & 12 o56'53"S & $38 \div 25^{\prime} 23^{\prime \prime} \mathrm{W}$ \\
\hline P2 & $12 \circ 57^{\prime} 09^{\prime \prime S}$ & $38 \div 25^{\prime} 09 " W$ \\
\hline P3 & $12 \div 57 ' 53 " \mathrm{~S}$ & $38 \div 24 ' 52 " W$ \\
\hline P4 & $12 \div 57^{\prime} 30 " \mathrm{~S}$ & $38 \div 25^{\prime} 12^{\prime \prime} \mathrm{W}$ \\
\hline P5 & $12 \div 57^{\prime} 24^{\prime \prime S}$ & $38 \div 25^{\prime} 39^{\prime \prime} \mathrm{W}$ \\
\hline P6 & 12 의'44"S & $38 \div 25^{\prime} 01^{\prime \prime} W$ \\
\hline P7 & $12 \div 57^{\prime} 07^{\prime \prime S}$ & $38 \div 24 ' 57 " W$ \\
\hline
\end{tabular}

As amostras de sedimento foram coletadas na estação chuvosa (agosto/2008). Para cada ponto, foram coletadas amostras de sedimento em triplicatas (cerca de 500 g cada), com auxílio de um testemunho, sendo retiradas em cada amostragem uma camada superficial de sedimento de $30 \mathrm{~cm}$. Em seguida, as amostras de sedimento foram colocadas individualmente em frascos de polietileno e armazenadas em freezer até a determinação dos metais. Todo material e vidraria usados nas análises foram previamente descontaminados $\left(\mathrm{HNO}_{3} 10 \%\right.$ por $\left.24 \mathrm{~h}\right)$.

\subsection{Preparo da amostra e determinação de metais}

As amostras de sedimento foram secas por 24 horas em temperatura ambiente, transferidas para o almofariz de porcelana, previamente limpo e seco. Em seguida, foram desagregadas, e homogeneizadas, quarteadas e peneiradas (10 mesh). As amostras foram digeridas segundo método USEPA-3051A (USEPA, 1998), no qual cerca de 0,5 g da amostra foi digerida em microondas (Provecto Analítica Sistemas DGT 100 PLUS) com $10 \mathrm{~mL}$ de $\mathrm{HNO}_{3} 14 \mathrm{~mol} / \mathrm{L}$ por 10 minutos, com a seguinte programação: $5 \mathrm{~min}(300 \mathrm{~W}) ; 5 \mathrm{~min}(500 \mathrm{~W}) ; 5 \mathrm{~min}(0 \mathrm{~W}) ; 5 \mathrm{~min}$ $(750 \mathrm{~W}) ; 5 \mathrm{~min}(0 \mathrm{~W})$. Após resfriamento natural das amostras digeridas, as mesmas foram filtradas (Whatman no 41), transferidas e avolumadas para um balão volumétrico de $50 \mathrm{~mL}$.

Para a validação do método empregado foi utilizado o padrão de referência certificado de STSD-4 da Mining and Mineral Sciences Laboratories (NRCan). 0 percentual de recuperação dos elementos foram: $\mathrm{Cu}(85,1 \%), \mathrm{Fe}(84,4 \%), \mathrm{Mn}(113,3 \%)$ e V $(87,1 \%)$. 
A determinação de metais ( $\mathrm{Al}, \mathrm{Cu}, \mathrm{Fe}, \mathrm{Mn}$ e $\mathrm{V}$ ) foi realizada utilizando a técnica da espectrometria de emissão óptica com plasma indutivamente acoplado (ICP OES), com o espectrômetro de plasma CIRUS CCD, simultâneo, de amostrador automático (auto sample Spectro) e nebulizador tipo crossflow (fluxo cruzado). O limite de detecção (LOD) do método foi calculado a partir da formula LOD = 3x/y, onde $x$ foi o desvio padrão de 20 amostras (branco) e y, é o coeficiente da curva analítica do ICP OES (SILVA et al., 2016). Os valores encontrados foram (mg $\left.\mathrm{kg}^{-1}\right)$ : Al $(0,0038), \mathrm{Cu}(0,0013), \mathrm{Fe}(0,0070), \mathrm{Mn}(0,0003)$ e V $(0,0010)$.

\subsection{Análises estatísticas}

A análise da significância entre as concentrações do material certificado recuperado (STSD4) e os seus valores de referência foi por meio do teste $t$-student. A diferença entre as variâncias nas concentrações dos metais nos pontos amostrais foi analisada pela Análise de Variância (ANOVA), seguida do teste de Tukey para os dados paramétricos ou pelo teste de Kruskal-Wallis seguido do teste de Mann-Whitney, quando os dados foram não paramétricos. A análise de correlação entre os pares de elementos foi por meio da Correlação de Spearman. Todas as análises foram realizadas utilizando o software SPSS 20.0 e considerando os níveis de probabilidade $p<0,01$.

\section{RESULTADOS E DISCUSSÕES}

As concentrações de todos os metais nos sedimentos analisados estão descritas na Tabela 2. Dentre os metais, o Fe e o Al apresentaram as maiores concentrações. Entre os pontos amostrais, o P1 e o P3 apresentaram, respectivamente, as maiores e menores concentrações para todos os metais.

Tabela 2: Concentração ( $\mathrm{mg} \mathrm{kg}^{-1} /$ peso seco) dos elementos químicos no sedimento da lagoa do Parque Metropolitano de Pituaçu, Salvador, Brasil.

\begin{tabular}{cccccc}
\hline $\begin{array}{c}\text { Ponto } \\
\text { Amostral }\end{array}$ & Al & Fe & Mn & V & Cu \\
\hline P1 & $26.672 \pm 861^{\mathrm{b}}$ & $122.030 \pm 5.604^{\mathrm{a}}$ & $481,50 \pm 94,05^{\mathrm{a}}$ & $75,00 \pm 32,53^{\mathrm{b}}$ & $23,00 \pm 2,83^{\mathrm{a}}$ \\
P2 & $16.230 \pm 2,07^{\mathrm{c}}$ & $35.976 \pm 2,45^{\mathrm{d}}$ & $227,00 \pm 5,66^{\mathrm{b}}$ & $75,00 \pm 11,31^{\mathrm{b}}$ & $17,00 \pm 1,41^{\mathrm{b}}$ \\
P3 & $12.043 \pm 1.076^{\mathrm{d}}$ & $17.210 \pm 314^{\mathrm{f}}$ & $60,50 \pm 31,82^{\mathrm{c}}$ & $25,00 \pm 5,66^{\mathrm{de}}$ & $\mathrm{Nd}$ \\
P4 & $4.633 \pm 329^{\mathrm{e}}$ & $11.968 \pm 763^{\mathrm{f}}$ & $44,00 \pm 29,70^{\mathrm{c}}$ & $40,00 \pm 21,21^{\mathrm{e}}$ & $4,05 \pm 2,62^{\mathrm{d}}$ \\
P5 & $3.618 \pm 1.096^{\mathrm{e}}$ & $82.893 \pm 9.741^{\mathrm{b}}$ & $89,00 \pm 8,49^{\mathrm{cd}}$ & $111,50 \pm 21,92^{\mathrm{a}}$ & $25,50 \pm 0,71^{\mathrm{a}}$ \\
P6 & $27.210 \pm 1.036^{\mathrm{a}}$ & $43.358 \pm 1.423^{\mathrm{c}}$ & $54,00 \pm 16,97^{\mathrm{c}}$ & $44,00 \pm 9,90^{\mathrm{ce}}$ & $5,80 \pm 1,56^{\mathrm{cd}}$ \\
P7 & $24.072 \pm 2.186^{\mathrm{b}}$ & $32.892 \pm 604^{\mathrm{e}}$ & $85,00 \pm 35,36^{\mathrm{cd}}$ & $65,00 \pm 9,90^{\mathrm{b}}$ & $9,70 \pm 3,25^{\mathrm{c}}$ \\
\hline
\end{tabular}

nd: não determinado.

Valores com letras minúsculas diferentes, para a mesma coluna, apresentaram diferenças significativamente a nível de $p<0,01$.

O Al apresentou concentrações que variaram de $3.618 \pm 1.096$ a $27.210 \pm 1.036 \mathrm{mg} \mathrm{Kg}^{-1}$, onde os pontos amostrais P1 e P6 apresentaram as maiores concentrações, entretanto, ambas foram estatisticamente similares. Valores semelhantes, mas com faixas de concentrações maiores foram encontrados por Vrhovnik et al., (2013) em sedimentos no lago Kalimanci, na Macedônia 
(5.720-101.100 mg kg-1) e por Abuduwaili et al., (2015) no lago Aibi, na China (18.900-65.200 mg $\left.\mathrm{kg}^{-1}\right)$.

O Fe apresentou valores entre $11.968 \pm 763$ a $122.030 \pm 5.604 \mathrm{mg} \mathrm{Kg}^{-1}$, com maiores concentrações nos pontos P1 e P5. A maioria dos pontos amostrais apresentaram valores similares aos encontrados por Franz et al., (2014), no Lago Paranoá, Brasil (38.730 mg kg ${ }^{-1}$ ), Sabino; Kastner; Amaral (2004), na Lagoa da Pampulha, Brasil (113.000 mg. $\mathrm{kg}^{-1}$ ) Sheela et al., (2012), no sedimento do lago Akkulam-Veli, na Índia (3.200-24.000 mg kg-1), Vrhovnik et al., (2013), em lago da Macedônia (41.900-112.700 mg kg-1) e por Schneider et al., (2014), no lago Macquarie, na Australia (2.000-27.000 $\left.\mathrm{mg} \mathrm{kg}^{-1}\right)$.

A concentração de $\mathrm{Mn}$ reportou grande variação entre os pontos amostrais $(44,00 \pm 29,70$ a 481,50 $\pm 94,05 \mathrm{mg} \mathrm{kg}^{-1}$ ) com as amostras da P1 apresentando valores cerca de 11 vezes maiores que a menor concentração encontrada (P4). Essa grande variabilidade e faixa de concentração também foram encontradas em sedimentos de outros lagos/lagoas brasileiros, tais como: no lago Paranoá, Brasília, com valores de 57,2 a 1.280,6 mg kg-1 (MAIA; BOAVENTURA; PIRES, 2006) e $68,57 \mathrm{mg} \mathrm{kg}^{-1}$ (FRANZ et al., 2014); na lagoa Rio das Pedras, com concentrações variando de 82,0 a $149 \mathrm{mg} \mathrm{kg}^{-1}$ (Tavares; Ferreira; Oliveira, 2003) e; na lagoa da Pampulha, Minas Gerais, com concentração de $360 \mathrm{mg} \mathrm{kg}^{-1}$ (Sabino; Kastner; Amaral, 2004). Em outras regiões do mundo também se observou essa tendência, onde Sheela et al., (2012), reporta variações de 0,0 - 276,0 mg.kg e Yao; Xue, (2014) Nanyihu, na China, faixas de 315 a $1.738 \mathrm{mg} \mathrm{kg}^{-1} \mathrm{de} \mathrm{Mn}$.

Os lagos são ambientes aquáticos que podem acumular metais no sedimento e com isso configurar como uma fonte amplificadora de poluição e disponibilizar os metais para os organismos (ABUDUWAILI et al., 2015). Assim, a concentração dos metais pesados no sedimento pode ser um indicador da poluição aquática revelando o status do sistema (YANG; LI; WANG, 2005). No caso do $\mathrm{Mn}$, altas concentrações no sedimento podem estar relacionadas com um ambiente aquático anóxico (CALVERT; PEDERSEN, 1996).

As concentrações encontradas para o $V$ foram entre $25,00 \pm 5,66$ e 111,50 $\pm 21,92 \mathrm{mg} \mathrm{Kg}^{-1}$, com maior concentração no ponto P5. Pesquisas na Lagoa Paranoá, também reportaram valores similares para o V, encontrando concentrações de 54,3 a 238,9 mg Kg${ }^{-1}$ (MAIA; BOAVENTURA; PIRES, 2006) e de $162,23 \mathrm{mg} \mathrm{Kg}^{-1}$ (FRANZ et al., 2014). Pesquisa de Khademi et al. (2015), em sedimentos do esturário Musa (Golfo Pérsia), reportou valores muito abaixo dos reportados nesta pesquisa (concentração média de $11,14 \mathrm{mg} \mathrm{kg}^{-1}$, peso seco).

Atualmente, o vanádio tem despertado interesse como foco de diversas pesquisas ambientais (DOBROWOLSKI; ADAMCZYK; OTTO, 2013). Ele é um metal utilizado em diversas atividades humanas e liberado em grande quantidade pela queima de combustíveis fósseis e vários processos industriais (KABATA-PENDIAS; MUKHERJEE, 2007). Pode ser encontrado em porfirina de petróleo e, por isso, pode ser utilizado como indicador de poluição aquática por petróleo (KHOSHNOOD et al., 2011).

Para o Cu observou-se variações de 4,05 $\pm 2,62$ a $25,50 \pm 0,71 \mathrm{mg} \mathrm{Kg}^{-1}$. Diversas pesquisas reportam faixas de concentrações similares para lagos de outras regiões do mundo (Tabela 3 ). Dentre os elementos investigados nesta pesquisa, o Cu é o único que apresenta valores limítrofes regulados por órgãos ou agências internacionais. Os valores encontrados estão dentro do limite tolerável pelo Ministry of Agriculture, Fisheries and Food (MAFF, 1998) e pela Environmental Protection Agency (USEPA, 1997) que estabelecem valores máximos de 40, 36 e $136 \mathrm{mg} \mathrm{kg}^{-1}$, respectivamente. 
Tabela 3 - Concentração de Cu encontrada no sedimento de diferentes lagos e regiões do mundo.

\begin{tabular}{|c|c|c|}
\hline Lago/Região & $\begin{array}{l}\text { Concentração } \\
\left(\mathrm{mg} \mathrm{kg}^{-1}\right)\end{array}$ & Referência \\
\hline Koumoundourou/Atenas, Grécia & $8,85-24,58$ & Hahladakis et al., (2013) \\
\hline Honghu/ China & $25-55$ & Hu et al., (2012) \\
\hline NortheasternPolish/ Polônia & 48,60 & $\begin{array}{l}\text { Tylmann et al., (2011) } \\
\text { López; Gierlowski- }\end{array}$ \\
\hline Hope/ VintonCounty, Ohio & 22,50 & $\begin{array}{c}\text { Kordesch; Hollenkamp, } \\
\text { (2010) }\end{array}$ \\
\hline Nanyihu/China & $14,9-38,5$ & Yao; Xue, (2014) \\
\hline Hulun/ China & $3,39-31,92$ & Weiping et al., (2015) \\
\hline Akkulam-Veli/ Kerala, India & $4,6-92,8$ & Sheela et al., (2012) \\
\hline Lake Macquarie/ Australia & $0,0-79,0$ & Schneider et al., (2014) \\
\hline Este estudo & $5,80-25,80$ & Silva et al., (2016) \\
\hline
\end{tabular}

Houve correlação positiva significativa entre pares dos elementos Al, Mn, V e Cu (Figura 3), com maiores coeficientes de determinação para correlações envolvendo o $\mathrm{Cu}\left(\mathrm{Al}-\mathrm{Cu}: \mathrm{R}^{2}=0,90\right.$; $\mathrm{V}$ $\left.\mathrm{Cu}: \mathrm{R}^{2}=0,99\right)$. O Fe, apesar de apresentar correlações mais fracas, reportou as seguintes associações significativas: Fe-Al $\left(R^{2}=0,57\right)$, Fe-Mn $\left(R^{2}=0,62\right)$, Fe-V $\left(R^{2}=0,63\right)$ e Fe-Cu $\left(R^{2}=0,67\right)$.

A correlação entre os elementos pode indicar que eles estão associados à geologia da área ou indicar que seus óxidos/hidróxidos podem ser um dos mecanismos de adsorção desses elementos (MAIA; BOAVENTURA; PIRES, 2006). 


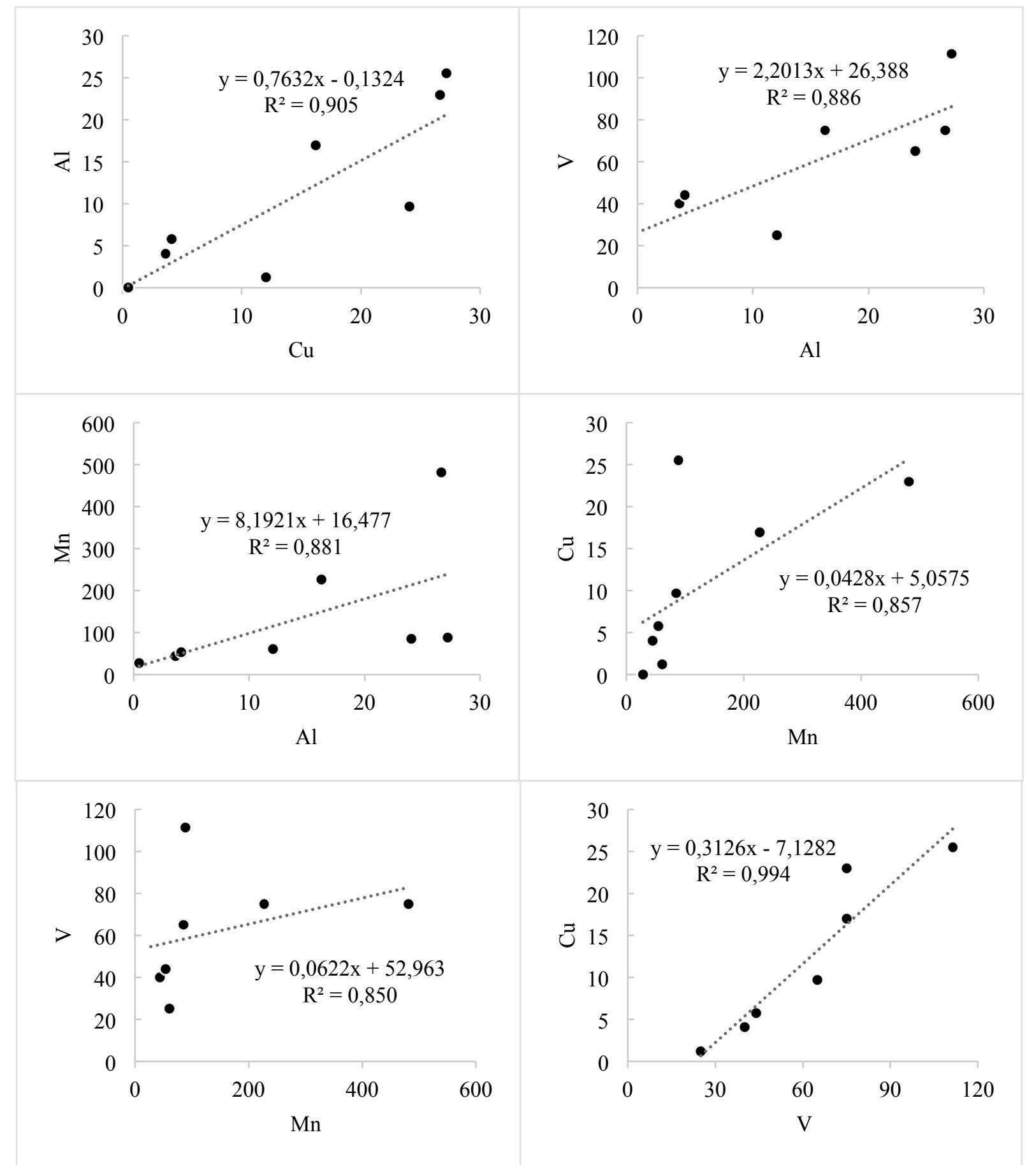

Figura 3 - Correlações entre pares de elementos químicos que apresentaram correlações significativas $(p<0,01)$.

\section{CONCLUSÃO}

Houve variações espaciais nas concentrações dos metais na Lagoa de Pituaçu, entretanto, os pontos amostrais que apresentaram maiores concentrações de metais foram os pontos P1, P2 e P5.

A concentração para a maioria dos metais investigados foi similar ou menor que aquelas reportadas para outros sedimentos de lagos brasileiros e de outras regiões do mundo, exceto para 
o vanádio, que todas as amostras investigadas apresentaram concentrações maiores que aquelas observadas na literatura. $\mathrm{O}$ acúmulo de vanádio no ambiente pode estar relacionado à fontes antrópicas, particularmente, da queima de combustíveis fósseis e de processos industriais.

Esse estudo indica que ampliações de ações de preservação e de monitoramento ambiental de contínuo fluxo de metais devem ser realizadas no ecossistema da Lagoa de Pituaçu, evitando, assim, a acumulação, em níveis tóxicos, de metais na água e no sedimento que comprometam à biota.

\section{REFERÊNCIAS}

ABUDUWAILI, J., ZHANG, Z. Y., \& JIANG, F. Q. (2015). Assessment of the distribution, sources and potential ecological risk of heavy metals in the dry surface sediment of Aibi lake in northwest China. PLOS ONE, 17(3), 1-16.

Al-Musharafi, S. K., Mahmoud, I. Y., \& Al-Bahry, S. N. (2014). Environmental Hazards and Pollution from Liquid Waste Lagoons. 5th International Conference on Environmental Science and Technology, 69, 1-5.

Calvert, S. E., \& Pedersen, T. F. (1996). Sedimentary geochemistry of manganese: implications for the environment of formation of manganiferous black shales. Economic Geology, 91(1), 36-47.

Couto, V. C. (2011). Análise Geoambiental da Bacia Hidrográfica do Rio Coxipó em Cuiabá/MT (1988, 2000 e 2010), Três Lagoas, 2011. Dissertação do Programa de Pós-graduação de Geografia, Três Lagoas: Universidade Federal de Mato Grosso do Sul.

Dobrowolski, R., Adamczyk, A., \& Otto, M. (2013) Determination of vanadium in soils and sediments by the slurry sampling graphite furnace atomic absorption spectrometry using permanent modifiers. Talanta, 113, 19-25.

Dural, M., Göksu, M. Z., \& Özak, A. A. (2007). Investigation of heavy levels in economically important fish species captured from the Tusla lagoon. Food Chemistry, 102(1), 415-421.

Fidan, A. F., Cigerci, I. H., Konuk, M., Küçükkurt, I., Aslan, R., \& Dündar, Y. (2008). Determination of some heavy metal levels and oxidative status in Carassius carassius L., 1758 from Eber Lake. Environmental Monitoring and Assessment, 147(1-3), 35-41.

Franz, C., Makeschin, F., Weib, H., \& Lorz, C. (2014). Sediments in urban river basins: Identification of sediment sources within the Lago Paranoá catchment, Brasilia DF, Brazil-using thefingerprint approach. Science of the Total Environment, 466-467(1), 513-523.

González, E., Ahumada, R., Medina, V., Neira, J., \& González, U. (2004). Espectrofotometría de absorción atómica con tubo en la llama: aplicación en la determinación total de cadmio, plomo y zinc en aguas frescas, agua de mar y sedimentos marinos. Química Nova, 27(6), 873-877. 
Hahladakis, J., Smaragdaki E., Vasilaki G., \& Gidarakos E. (2013). Use of Sediment Quality Guidelines and pollution indicators for the assessment of heavy metal and PAH contamination in Greek surficial sea and lake sediments. Environmental Monitoring Assessment, 185(3), 2843-2853.

HU, Y., Qi, S., Wu, C., Ke, Y., Chen, J., Chen, W., \& Gong, X. (2012). Preliminary assessment of heavy metal contamination in surface water and sediments from Honghu Lake, East Central China. Frontiers of Earth Science, 6(1), 39-47.

Kabata-Pendias, A., \& Mukherjee, A. B. (2007). Trace Elements from Soil to Human. 1st. SpringerVerlag $\mathrm{GmbH}$, Berlin, Heidelberg, New York.

KHADEMI, N. Riyahi-Bakhtiari, A., Sobhanardakani, S., Rezaie-Atagholipour, M., \& Burger, J. (2015). Developing a bioindicator in the Northwestern Persian Gulf, Iran: trace elements in bird eggs and in coastal sediments. Archives of Environmental Contamination and Toxicology, 68(2), 274-282.

Khoshnood, Z., Mokhlesi, A., \& Khoshnood, R. (2011). Effects of oil pollution on EURYGLOSSA ORIENTALIS and PSETTODES ERUMEI in the Persian Gulf. Romeno Journal of Biologia e Zoologia, 56(2), 201-209.

López, D. L., Gierlowski-Kordesch, E., \& Hollenkamp, C. (2010). Geochemical Mobility and Bioavailability of Heavy Metals in a Lake Affected by Acid Mine Drainage: Lake Hope, Vinton County, Ohio. Water Air and Soil Pollution, 213(1), 27-45.

Ministry of Agriculture, Fisheries, Food [MAFF]. Code of good agricultural practice for the protection of water. MAFF Publications, London, 1998.

Maia, P. D., Boaventura, G. R., \& Pires, A. C.B. (2006). Distribuição espacial de elementos-traço em sedimentos do lago Paranoá - DF, Brasil. Geochimica Brasiliensis, 20(2), 158-174.

Meucci, V., Laschi, S., Minunni, M., Pretti, C., Intorre, L., \& Soldani, G. (2009). An optimized digestion method coupled to electrochemical sensor for the determination of $\mathrm{Cd}, \mathrm{Cu}, \mathrm{Pb}$ and $\mathrm{Hg}$, in fish by square wave anodic stripping voltametry. Talanta, 77(3), 1143-1148.

Montanha, F. P., Astrauskas, J. P., Kirnew, M. D., Nagashima, J. C., \& Pimpão, C. T. (2011). Degradação de ambientes aquáticos por exposição a compostos químicos. Revista Científica Eletrônica de Medicina Veterinária, 10(17), 1-12.

Pompeo, M. L. M., Padial, P. R., Mariani, C. F., Cardoso-Silva, S., Moschini-Carlos, V., Silva, D. C. V. R. P., Paiva, T. C. B., \& Brandimarte, A. L. (2013). Biodisponibilidade de metais no sedimento de um reservatório tropical urbano (reservatório Guarapiranga - São Paulo (SP), Brasil): há toxicidade potencial e heterogeneidade espacial? Geochimica Brasiliensis, 27(2), 104-119.

Porto, M. F. A; \& Porto, R. L. L. (2008). Gestão de bacias hidrográficas. Estudos Avançados, 22(63), 43-60. 
Sabino, C. V. S., Kastner, G. F., \& Amaral, A. M. (2004). Estudo da biodisponibilidade de metais nos sedimentos da lagoa da Pampulha. Química Nova, 27(2), 231-235.

SANTANA, M. S. (2003). Experimento de Produção Primária no Reservatório de Pituaçu, Salvador, 2003. Monografia do Curso de Ciências Biológicas, Salvador: Universidade Federal da Bahia.

Schneider, L. Maher, W., Potts, J., Gruber, B., Batley, G., Taylor, A., Chariton, A., Krikowa, F., Zawadzki, A., \& Heijnis, H. (2014). Recent history of sediment metal contamination in Lake Macquarie, Australia, and an assessment of ash handling procedure effectiveness in mitigating metal contamination from coal-fired power stations. Science of the Total Environment, 490, 659670.

SCHRIKS, M., Heringa, M. B., Van der Kooi, M. M., Voogt, P., \& Van Wezel, A. P. (2010). Toxicological relevance of emerging contaminants for drinking water quality. Water Research, 44(2), 461-76.

Secretaria do Meio Ambiente - Bahia [SEMA]. Disponível em: https://www2.meioambiente.ba.gov.br.

Sheela, A. M., Letha, J., Joseph, S., \& Thomas, J. (2012). Assessment of heavy metal contamination in coastal lake sediments associated with urbanization: Southern Kerala, India. Lakes \& Reservoirs: Research and Management, 17(2), 97-112.

Silva, E. Viana, Z. C. V., Souza, N. F. A., Korn, M. G. A., \& Santos, V. L. C. S. (2016). Assessment of essential elements and chemical contaminants in thirteen fish species from the Bay Aratu, Bahia, Brasil. Brazilian Journal of Biology, 76(3), 871-877.

Souza, V. L. B., Lima, V. L., Hazin, C. A., Fonseca, C. K. L., \& Santos, S. O. (2015). Biodisponibilidade de metais-traço em sedimentos: uma revisão. Brazilian Journal of Radiation Sciences, 3(1a) ), 1-13.

Tavares, G. A., Ferreira, J. R., \& Oliveira, C. R. (2003). Histórico da presença de metais pesados (Hg, $\mathrm{Cu}, \mathrm{Mn}, \mathrm{Cr}$ e Ni) na lagoa Rio das Pedras, Bacia do Rio Moji-Guaçu - SP, Brasil. Holos Environment, 3(2), 74-84.

Tylmann, W., Łysek, K., Kinder, M., \& Pempkowiak, J. (2011). Regional pattern of heavy metal content in Lake sediments in Northern Poland. Water Air and Soil Pollution, 216(1-4), 217-228.

Tucci, C. E. M. (1997). Hidrologia: ciência e aplicação. 2.ed. Porto Alegre: ABRH/Editora da UFRGS, 1997.

United States Environmental Protection Agency [U.S. EPA]. (1998). Method 3051a - microwave assisted acid digestion of sediments, sludges, soils, and oils. Disponível em: https://www.epa.gov/sites/production/files/2015-12/documents/3051a.pdf. 
United States Environmental Protection Agency [U.S. EPA]. (1997). Exposure Factors Handbook (1997 Final Report). U.S. Environmental Protection Agency, Washington, DC, EPA/600/P-95/002F a-c, $1997 . \quad$ Disponível em: https://www3.epa.gov/airtoxics/cancer_guidelines_final_3-25-05.pdf.

Vrhovnik, P., S`muc, N. R., Dolenec, T., Serafimovski, T., \& Dolenec, M. (2013). An evaluation of trace metal distribution and environmental risk in sediments from the Lake Kalimanci (FYR Macedonia). Environmental Earth Sciences, 70(2), 761-775.

Weiping, L., Zhang, W. B., \& Yu, J. P. (2015). Distribution features and ecological risk assessment of heavy metals in superficial sedments of Hulun Lake. Journal of Applied Sciences, 15(4), 682-688.

Yang, Z., Li, G. \& Wang, D. (2005). The assessment of heavy metals of Baiyang lake sediment and its potential ecological risk. Agriculture Environmental Science, 24, 945-951.

Yao, S., \& Xue, B. (2014). Heavy metal records in the sediments of Nanyihu Lake, China: influencing factors and source identification. Journal of Paleolimnology, 51, 15-27. 